\title{
Distribution Network Reconfiguration Using Binary Particle Swarm Optimization to Minimize Losses and Decrease Voltage Stability Index
}

\author{
Aji Akbar Firdaus ${ }^{1}$, Ontoseno Penangsang ${ }^{2}$, Adi Soeprijanto ${ }^{3}$, Dimas Fajar U. P. ${ }^{4}$ \\ ${ }^{1}$ Department of Engineering, Faculty of Vocational, Universitas Airlangga \\ Gubeng, Surabaya 60286, Indonesia \\ ${ }^{2,3,4}$ Department of Electrical Engineering, Institut Teknologi Sepuluh Nopember \\ Sukolilo, Surabaya 60111, Indonesia
}

\begin{abstract}
Article Info
Article history:

Received Oct 11, 2017

Revised Aug 26, 2018

Accepted Oct 25, 2018

\section{Keywords:}

BIBC-BCBV

BPSO

Losses

Network reconfiguration VSI

ABSTRACT

Power losses and voltage drop are existing problems in radial distribution networks. This power losses and voltage drop affect the voltage stability level. Reconfiguring the network is a form of approach to improve the quality of electrical power. The network reconfiguration aims to minimize power losses and voltage drop as well as decreasing the Voltage Stability Index (VSI). In this research, network reconfiguration uses binary particle swarm optimization algorithm and Bus Injection to Branch Current-Branch Current to Bus Voltage (BIBC-BCBV) method to analyze the radial system power flow. This scheme was tested on the 33-bus IEEE radial distribution system $12.66 \mathrm{kV}$. The simulation results show that before reconfiguration, the active power loss is $202.7126 \mathrm{~kW}$ and the VSI is 0.20012 . After reconfiguration, the active power loss and VSI decreased to $139.5697 \mathrm{~kW}$ and 0.14662 , respectively. It has decreased the power loss for $31.3136 \%$ significantly while the VSI value is closer to zero.
\end{abstract}

Copyright () 2018 Institute of Advanced Engineering and Science. All rights reserved.

\section{Corresponding Author:}

Aji Akbar Firdaus,

Department of Engineering,

Faculty of Vocational, Universitas Airlangga

Gubeng, Surabaya 60286, Indonesia

Email: aji.akbar.firdaus11@gmail.com

\section{INTRODUCTION}

The distribution of electrical power from the substation to load requires distribution lines. There are several problems with the distribution system, such as losses and voltage drop. Losses and voltage drop are affected by the main parameters of the resistance and reactance current in feeders [1]. Therefore, network reconfiguration method in the distribution system is applied to overcome the problems.

The reconfiguration of the distribution network is one of the available approaches to reduce losses in the distribution network and improve the reliability of the distribution system. Reconfiguration is processed by altering the topological structure of distribution networks through the open and close status of the sectionalizers and tie switches in the distribution system [2].

In general, the reconfiguration of a distribution network can be classified into two groups, those are heuristic and artificial intelligence (AI). Reconfiguration of the distribution network by using a heuristic approach proposed a heuristic switch exchange algorithm to reduce losses and presented a simple formula to estimate changes in power losses from a feeder to another [3]. In other research, another formula was introduced as a calculation for the load balancing index [4]. The heuristic method was also developed [5] considering all switches closed and the switch opened to form a radial network. 
Several AI-based approaches using various types of meta-heuristics and evolutionary algorithm have been proposed for the optimization of the distribution network reconfiguration. Those approaches cover reconfiguration of distribution network using Genetic Algorithm, Binary Genetic Algorithm, and Particle Swarm Optimization to reduce power losses [6-8]. The implementation of several AI methods previously mentioned was also developed in Distributed Generation (DG) [9, 10]. The implementation of several AI methods has increased the power quality and Load Balancing Index (LBI) based on PSO and BPSO methods $[11,12]$. However, only a few papers have discussed VSI.

In this study, the distribution network reconfiguration is implemented to minimize losses and improve system stability in the distribution networks based on BPSO algorithm to solve discrete function in optimization problems. The discrete function is the selection of any combination provided by the switches from the distribution network. In this research, the power flow in the radial distribution system could be calculated with Bus Injection to Branch Current-Branch Current to Bus Voltage (BIBC-BCBV) method. The level of voltage stability for the distribution system is determined by the Voltage Stability Index (VSI). The appropriate reconfiguration could be decided after considering the index value. It depends on the closeness to the unity, the closer the feasible candidates to the unity the better the results. The next section of this paper is organized as follows: Section 2 describes the methodology of the proposed method. The simulation results of the voltage profile, minimal losses, switches selection, and voltage stability index are presented and supported by analysis in section 3 . Finally, in section 4 , the conclusions are obtained from the results and the analysis.

\section{DESCRIPTION OF THE PROPOSED METHOD}

\subsection{Distribution Network Reconfiguration Problem Formulation}

This paper has two objectives those are losses minimization and stability improvement of the system. Minimal losses are able to increase the system economical value. Moreover, it could be obtained by reconfiguring the system. The Voltage Stability Index (VSI) is an important parameter to the system stability. The first objective function is to minimize the total loss by following (1) and (2) [13]. The symbol of Ploss and Qloss are the total active and reactive power loss, respectively. $B B(j j)$ is the branch current. The symbol $R(j j)$ and $X(j j)$ are the resistance and the inductance.

$$
\begin{aligned}
& \text { Ploss }=\left|B B(j j)^{2}\right| R(j j) \\
& \text { Qloss }=\left|B B(j j)^{2}\right| X(j j)
\end{aligned}
$$

The second objective function is VSI. VSI is used to determine the stability of the distribution system. The distribution system models are converted into two buses system [14]. Figure 1 shows an equivalent of the two buses system while VSI equation is shown in (5). Voltage stability level of the total interconnected system could be measured by using the proposed VSI. In this model, the system is declared to be stable if the value of the index is closer to zero [15]. $P_{i+1}$ and $Q_{i+1}$ are the total active and reactive power from the total load. $V_{i+1}$ is voltage receiving. In addition to that, $r_{i}$ and $x_{i}$ are equivalent circuit model. Those are obtained by calculating the total active and reactive power loss in (3) and (4). The symbol of $P_{l}$ and $Q_{l}$ are the total active and reactive power generation. And $V_{i}$ is voltage sending.

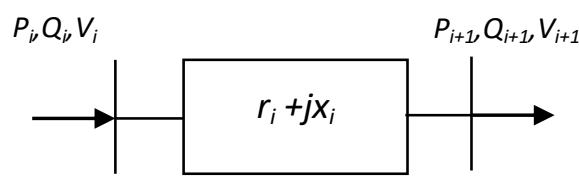

Figure 1. The equivalent of two buses system

$$
\begin{aligned}
& P_{l}=r_{i} \frac{\left(P_{i+1}^{2}+Q_{i+1}^{2}\right)}{V_{i+1}^{2}} \\
& Q_{l}=x_{i} \frac{\left(P_{i+1}^{2}+Q_{i+1}^{2}\right)}{V_{i+1}^{2}}
\end{aligned}
$$


The value of VSI must be less than 1

$$
\left(L_{i}\right)=\frac{\sqrt[4]{\left(P_{i+1}^{2}+Q_{i+1}^{2}\right) \cdot\left(r_{i}^{2}+x_{i}^{2}\right)}}{V_{i}} \leq 1
$$

\subsection{Bus Injection to Branch Current-Branch Current to Bus Voltage (BIBC-BCBV) Method}

Power flow in a distribution system is different from the power flow in a transmission system because a distribution system has a radial network [16]. One of the methods to calculate power flow in the radial distribution system is Bus Injection to Branch Current-Branch Current to Bus Voltage (BIBC-BCBV). Figure 2 shows a simple example of the radial distribution network. The symbols of B1 until B5 are branches current from line 1 until line 5 . $I_{1}$ to $I_{6}$ are the current injection from bus 1 until bus 6 .

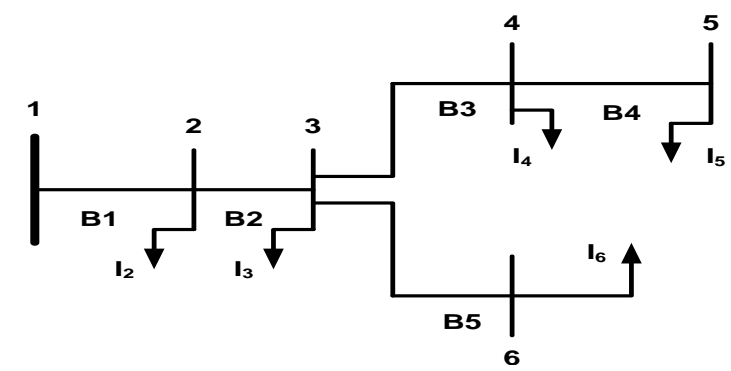

Figure 2. Example of a radial distribution network

From Figure 2, the current injection presented in the BIBC matrix as shown as in (6).

$$
\begin{aligned}
& {\left[\begin{array}{l}
B_{1} \\
B_{2} \\
B_{3} \\
B_{4} \\
B_{5}
\end{array}\right]=\left[\begin{array}{lllll}
1 & 1 & 1 & 1 & 1 \\
0 & 1 & 1 & 1 & 1 \\
0 & 0 & 1 & 1 & 0 \\
0 & 0 & 0 & 1 & 0 \\
0 & 0 & 0 & 0 & 1
\end{array}\right]\left[\begin{array}{l}
I_{2} \\
I_{3} \\
I_{4} \\
I_{5} \\
I_{6}
\end{array}\right]} \\
& {[B]=[B I B C][I]}
\end{aligned}
$$

BCBV matrix is obtained from the voltage drop in (8).

$$
\begin{aligned}
& {\left[\begin{array}{l}
V_{1}-V_{2} \\
V_{1}-V_{3} \\
V_{1}-V_{4} \\
V_{1}-V_{5} \\
V_{1}-V_{6}
\end{array}\right]=\left[\begin{array}{ccccc}
Z_{12} & 0 & 0 & 0 & 0 \\
Z_{12} & Z_{23} & 0 & 0 & 0 \\
Z_{12} & Z_{23} & Z_{34} & 0 & 0 \\
Z_{12} & Z_{23} & Z_{34} & Z_{45} & 0 \\
Z_{12} & Z_{23} & 0 & 0 & Z_{36}
\end{array}\right]\left[\begin{array}{l}
B_{1} \\
B_{2} \\
B_{3} \\
B_{4} \\
B_{5}
\end{array}\right]} \\
& {[\Delta V]=[B C B V][B]}
\end{aligned}
$$

If (7) and (9) substituted into the mentioned equation, subsequently the equation $\Delta \mathrm{V}$ transformed into (10) and (11).

$$
\begin{aligned}
& {[\Delta V]=[B C B V][B I B C][I]} \\
& {[\Delta V]=[D L F][I]}
\end{aligned}
$$

Completion of power flow computation could be obtained by iteration of (12) and (13).

$$
\begin{aligned}
& I_{i}^{(k)}=\left(\frac{P i+j Q i}{V i^{(k)}}\right)^{*} \\
& {\left[\Delta \mathrm{V}^{\mathrm{k}}\right]=[\mathrm{DLF}]\left[\mathrm{I}^{\mathrm{k}}\right]}
\end{aligned}
$$

As shown in (14) is used to update the new voltage for the next iterative steps 


$$
\left[\mathrm{V}^{\mathrm{k}+1}\right]=\left[\mathrm{V}_{1}\right]-\left[\Delta \mathrm{V}^{\mathrm{k}}\right]
$$

\subsection{The Particle Swarm Optimization}

The standard form of PSO algorithm could be written as in (15), and (16) [17,18]. The symbol of $v_{i}$ and $x_{i}$ are the velocity and the position of particles, successively. In this case, $c_{1}$ and $c_{2}$ are positive constants; $\varphi_{1}$ and $\varphi_{2}$ are two random variables occurred between 0 and $1 ; p_{i}$ is a combination of switches obtained from the best position of the particle; $p_{g}$ is a combination of switches obtained from the best position of all existing populations; $w$ is the inertia weight which states the effect of the previous velocity vector of the new vector.

$$
\begin{aligned}
& v_{i}(t+1)=w \cdot v_{i}(t)+c_{1} \varphi_{1}\left(p_{i}-x_{i}(t)\right)+c_{2} \varphi_{2}\left(p_{g}-x_{i}(t)\right) \\
& x_{i}(t+1)=x_{i}(t)+v_{i}(t+1)
\end{aligned}
$$

The standard procedure for applying the PSO algorithm is as follows:

a. Initialization of velocity, position, and parameters of the PSO

b. Update the particle velocity based on (15)

c. Update the particle position using (16)

d. Evaluation of the fitness function to update the value of $p_{i}$ and $p_{g}$

e. Compare each candidate of $p_{g}$ in the value of the fitness function in order to get the best value of $p_{g}$.

f. If the value of $p_{g}$ is not the best value, then return to step 2

g. Repeat the iterations until it reached the limit to get a $p_{g}$ with the smallest fitness function or the best value [17].

\subsection{Binary Particle Swarm Optimization}

The switches combination in this problem is the positive integers. Accordingly, the PSO algorithm requires next steps to convert the calculated results of the velocity and the position into positive integers such as in (17) and (18) [19]. The symbol of $v_{i j}$ and $x_{i j}$ refers to the velocity and the position of the particle. While $r_{i j}$ is a random number.

$$
\begin{aligned}
& v_{i j}(t)=\operatorname{sig}\left(v_{i j}(t)\right)=\frac{1}{1+e^{-v_{i j}(t)}} \\
& x_{i j}(t+1)=\left\{\begin{array}{lr}
1 \text { if } r_{i j}<\operatorname{sig}\left(v_{i j}(t+1)\right) \\
0 & \text { otherwise }
\end{array}\right.
\end{aligned}
$$

The modification procedure for applying the BPSO algorithm, in this case, is as follows:

a. Initialization of the velocity, the position, and the parameters of PSO

b. Update the particle velocity based on (15), then the result of (15) is used by (17)

c. Update the particle position using (18).

d. Evaluation of the fitness function to update $p_{i}$ and $p_{g}$

e. Compare each candidate of $p_{g}$ by the value of the fitness function in order to get the best value $p_{g}$.

f. If the result of $p_{g}$ is not the best available value and return to step 2

g. Repeat the iterations until it achieved the limit to get the value of $p_{g}$ with the smallest fitness function or the best value [17]. The fitness function or the best from (1) and (5) are the losses and the VSI.

\subsection{Genetic Algorithm (GA)}

The conventional GA is used to get minimal losses [7]. The standard procedure for applying GA, in this case, is similar to conventional GA. However, the VSI calculation is added in this paper.

\section{EXPERIMENTAL RESULTS}

The proposed reconfiguration method has been tested on the 33 buses system at $12.66 \mathrm{kV}$. This system consists of five tie-switches which are set to normally open and 32 sectionalizer switches that normally closed. Figure 3 is a schematic diagram of the test system. The original system is simulated in MATLAB by using the BIBC-BCBV method. Initially, the first opened switches of this system are switch$33(\mathrm{~s} 33),(\mathrm{s} 34),(\mathrm{s} 35),(\mathrm{s} 36)$, (s37). The initial power loss of this system is $202.6845 \mathrm{~kW}$, while the minimum voltage is $0.9131 \mathrm{pu}$, and the voltage stability condition of this system is at 0.20688 . The minimum voltage occurs at bus 33 because the furthest load is located on this bus. This result shows that the further the load is, the lower the voltage due to the increased losses. 


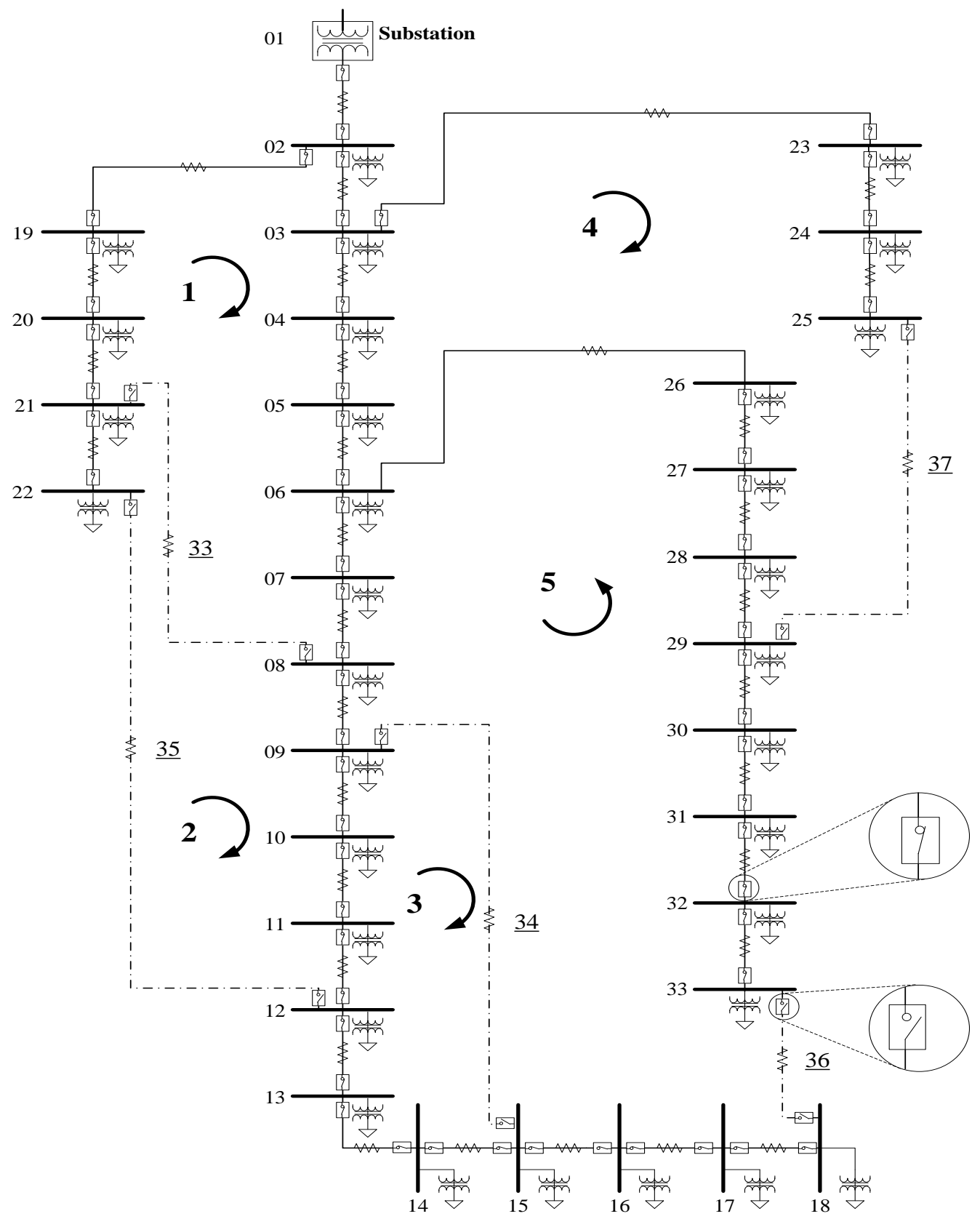

Figure 3. Schematic system 33 buses

The proposed reconfiguration method is BPSO. The parameters used in the simulation network are 20 populations, 5 dimensions, and 100 number of iterations. Figure 4 is the schematic diagram of the reconfiguration system using BPSO. After reconfiguration, the simulation results are obtained in Figure 5. The active and the reactive power losses calculated by using BIBC-BCBV method are 139.2168 kW and $102,0619 \mathrm{kVAR}$. The minimum voltage is $0.9378 \mathrm{pu}$ which is occurred on bus 32 . The percentage of power loss reduction is $31.3136 \%$. After the reconfiguration is processed, the voltage becomes better and the power loss compared to the initial condition is decreased. Figure 5 shows that there are 7 buses which the voltages are below $0.95 \mathrm{pu}$, as the minimum standard. Those buses are bus 17 to bus 18 and bus 29 to bus 33. This condition indicates that most buses before the reconfiguration processed were already in under-voltage condition and it has been fixed by reconfiguring the network. The quality of the electricity service to the consumers is increased with the average voltage obtained in the simulation is $0.9652 \mathrm{pu}$. The corresponding value of VSI for new configuration is 0.14999 . Thus, the total voltage stability level of the 33-bus system in 
the condition after reconfiguration is closer to zero than the initial VSI value. The final VSI value in Table 1 indicates better system stability.

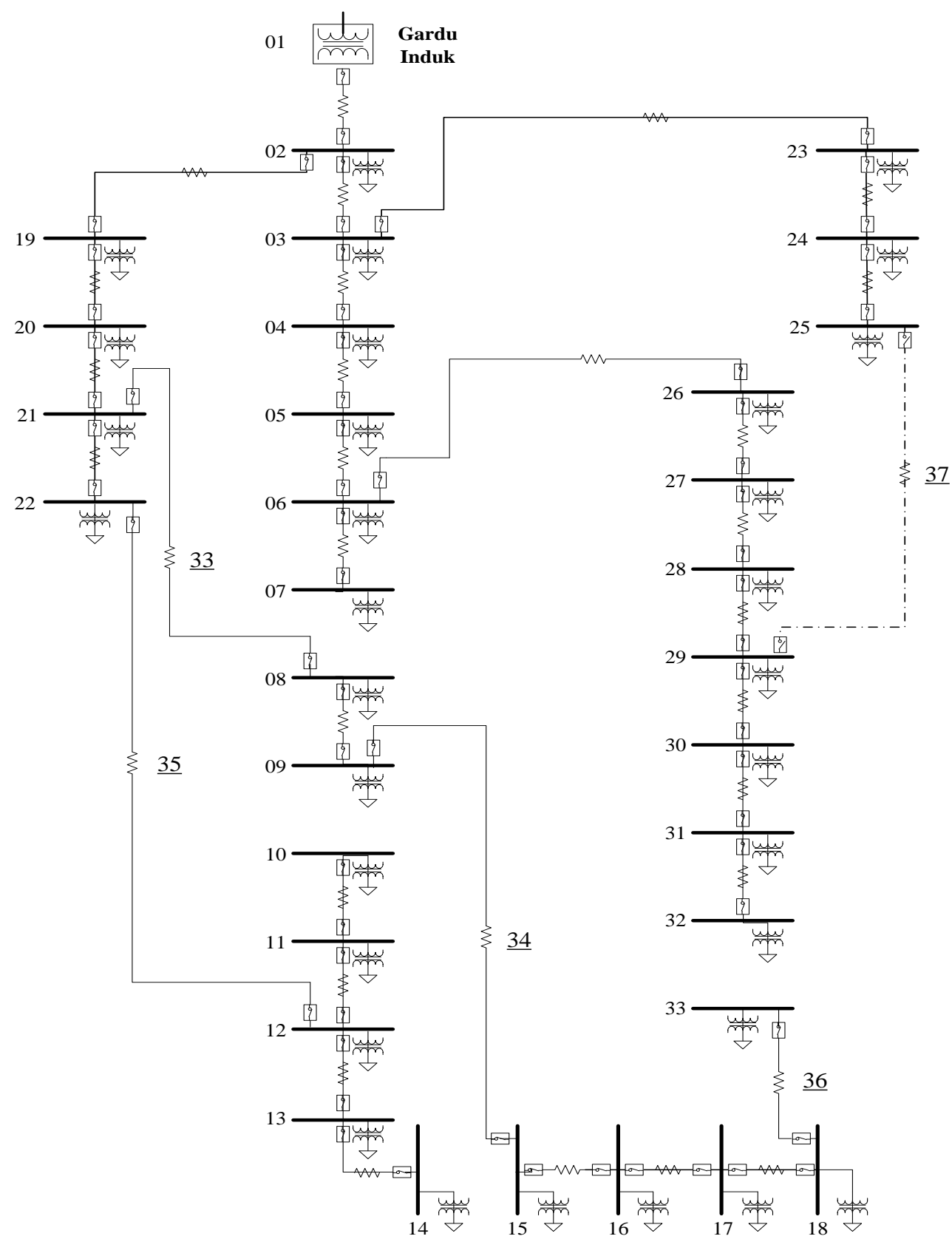

Figure 4. Schematic diagram of the reconfiguration system using BPSO

Table 1. Schematic System 33 Buses before Reconfiguration and after Reconfiguration with BPSO

\begin{tabular}{ccc}
\hline Scenario & & \\
\hline Before reconfiguration & Switch open & $33,34,35,36,37$ \\
& $P_{\text {loss }}(\mathrm{kW})$ & 202,6845 \\
& Minimum voltage $(\mathrm{pu})$ & 0,9131 \\
After reconfiguration & $L$-index & 0,20688 \\
& Switch open & $7,9,14,32,37$ \\
& $P_{\text {loss }}(\mathrm{kW})$ & 139,2168 \\
& Minimum voltage $(\mathrm{pu})$ & 0,9378 \\
& Loss reduction $(\%)$ & 31,3136 \\
& $L$-index & 0,14999 \\
\hline
\end{tabular}




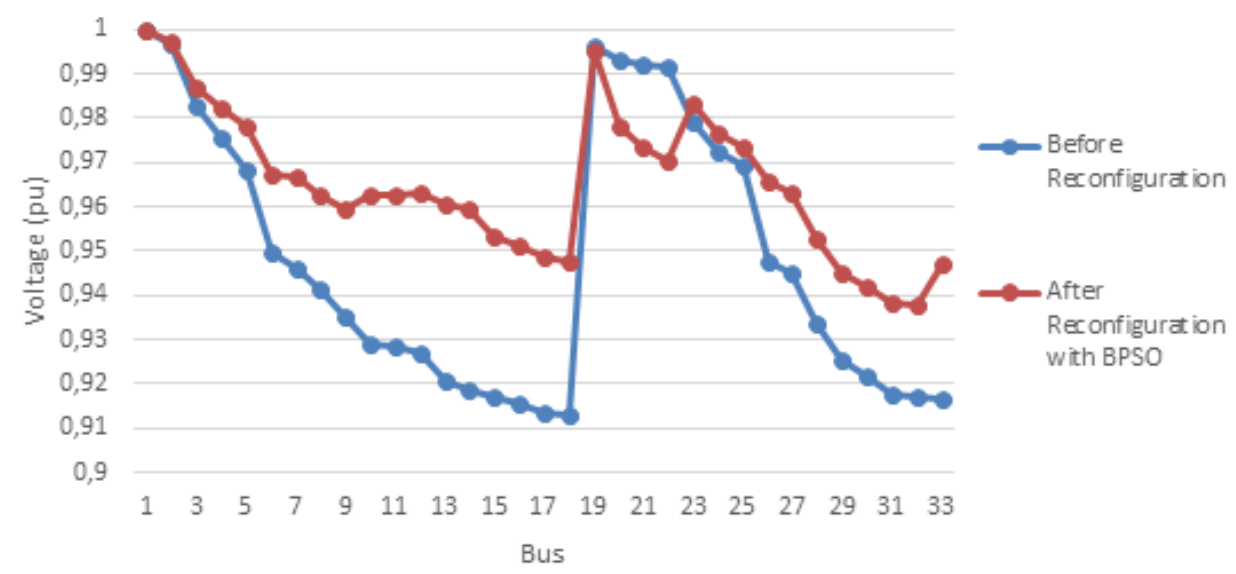

Figure 5. Voltage on each bus before and after the reconfiguration with BPSO

To compare the performance of BPSO, all scenarios are simulated with GA and the results are provided in Table 2. Reconfiguring a network using GA gives $146.4 \mathrm{~kW}$ of active power. The percentage of that power loss reduction is $27.7695 \%$. The minimum voltage on bus 33 is $0.937 \mathrm{pu}$. Figure 6 shows there are 6 buses below $0.95 \mathrm{pu}$. Those buses are bus 18, and bus 29 to bus 33. The average produced voltage of a network reconfiguration with GA is $0.96657 \mathrm{pu}$. The results of VSI value is 0.1531 . In Table 2, it is confirmed that the performance of BPSO is better than GA in the terms of quality of the solutions in all scenarios. The power losses from BPSO and GA are $139.2168 \mathrm{~kW}$ and $146.4 \mathrm{~kW}$, respectively. The value of VSI simulation results of BPSO and GA are 0.14999 and 0.1531.

Table 2. Comparison Schematic System 33 buses after the Reconfiguration with BPSO and after the Reconfiguration with GA

\begin{tabular}{ccc}
\hline Method & Item & After reconfiguration \\
\hline BPSO & Switch open & $7,9,14,32,37$ \\
& $P_{\text {loss }}(\mathrm{kW})$ & 139,2168 \\
& Minimum voltage (pu) & 0,9378 \\
& Loss reduction $(\%)$ & 31,3136 \\
& L-index & 0,14999 \\
$\mathrm{GA}$ & Switch open & $9,28,33,34,36$ \\
& $P_{\text {loss }}(\mathrm{kW})$ & 146,4 \\
& Minimum voltage $(\mathrm{pu})$ & 0,9370 \\
& Loss reduction $(\%)$ & 27,7695 \\
& $L$-index & 0,1531 \\
\hline
\end{tabular}

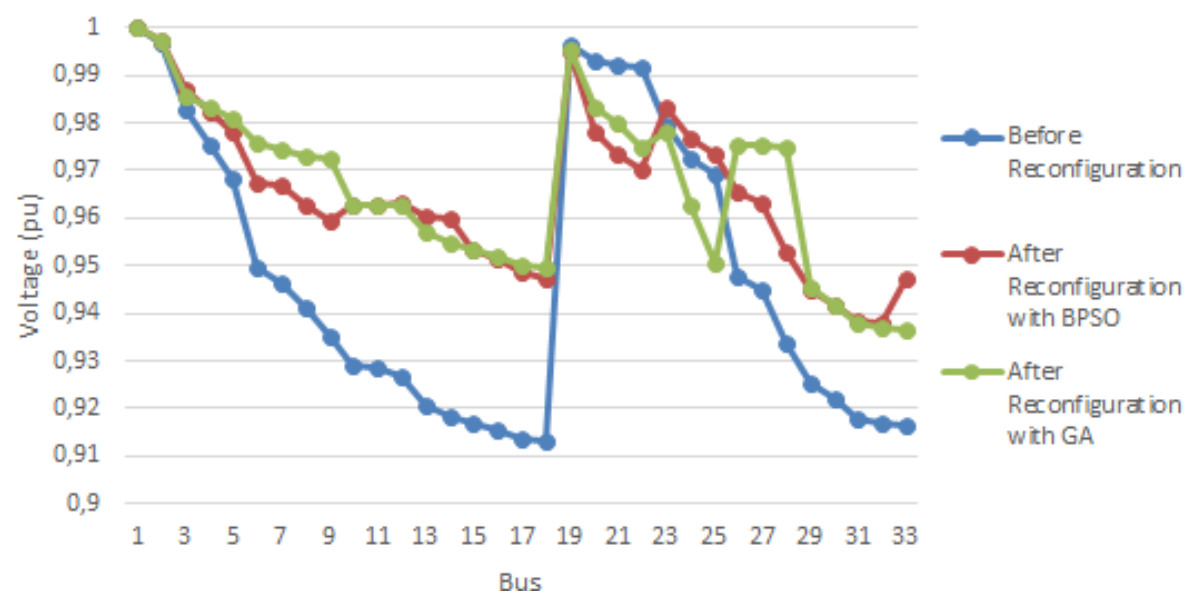

Figure. 6 The voltage profile each bus obtained from the calculation based on BPSO and GA 


\section{CONCLUSION}

The reconfiguration process is successfully conducted by using BPSO, which provides lower power losses and better VSI. The VSI value is closer to zero. The optimal reconfiguration is obtained when the opening switches are (s7), (s9), (s14), (s32), (s37). The simulation results from BPSO give a better minimal loss and VSI value than GA.

\section{REFERENCES}

[1] M.E. Baran and F. Wu, "Network reconfiguration in distribution system for loss reduction and load balancing", IEEE Trans. on Power Delivery, 1989, vol.4, no.2, pp. 1401-1407.

[2] Venkatesh et al, 2004. Optimal reconfiguration of radial distribution system to maximize loadability. IEEE Trans. on Power System, Vol. 19, No. 1, pp. 260-266.

[3] Civanlar, S., Grainger, J.J., Yin, H., Lee, S.S.H.: 'Distribution feeder reconfiguration for loss reduction', IEEE Trans. Power Deliv., 1988, 3, (3), pp. 1217-1223.

[4] Baran, M.E., Wu, F.F.: 'Network reconfiguration in distribution systems for loss reduction and load balancing', IEEE Trans. Power Deliv., 1989, 4, (2), pp. 1401-1407.

[5] Gomes, F.V.: 'A new distribution system reconfiguration approach using optimum power flow and sensitivity analysis for loss reduction', IEEE Trans. Power Syst., 2006, 21, (4), pp. 1616-1623.

[6] Nara, K., Shiose, A., Kiagawa, M., Ishihara, T.: 'Implementation of genetic algorithm for distribution system loss minimum configuration', IEEE Trans. Power Syst., 1992, 7, (3), pp. 1044-1051.

[7] Zhu, J.Z., "Optimal reconfiguration of electrical distribution network using the refined genetic algorithm", Electr. Power Syst. Res., 2002, 62, (1), pp. 37-42.

[8] Olimaei, J., Niknam, T., Gharehpetian, G.: "Application of particle swarm optimization for distribution feeder reconfiguration considering distributed generator", Appl. Math. Comput., 2008,201, (1-2), pp. 575-586.

[9] Mohammad Salehi Male, Adel Akbari Majd, Ramtin Rasouli Nezhad, "Optimal Determination of Size and Site of DGs in Mesh System Using PSO”, Bulletin of Electrical Engineering and Informatics (BEEI), Vol. 3, No. 2, June 2014, pp. 101 108.

[10] Ch.V.S.S. Sailaja, P.V.N. Prasad, "Reliabilty and Cost Benefit Analysis of DG Integrated Distribution System", Bulletin of Electrical Engineering and Informatics, Vol. 5, No. 4, December 2016, pp. 383 389.

[11] Mohammad Reza Andervazh, Javad Olamaei, Mahmoud Reza Haghifam, "Adaptive multi-objective distribution network reconfiguration using multi-objective discrete particles swarm optimisation algorithm and graph theory", IET Gener. Transm. Distrib., 2013, Vol. 7.

[12] Xiaoling Jin., Jianguo Zhao., Ying Sun., Kejun Li., Bqin Zhang., "Distribution Network Reconfiguration for Load Balancing Using Binary Particle Swarm Optimization", International Conference on Power System TechnologyPOWERCON, November 2004.

[13] G. W. Chang, S. Y. Chu, ” An Improved Backward/Forward Sweep Load Flow Algorithm for Radial Distribution Systems”, IEEE Transactions On Power Systems, Vol. 22, No. 2, May 2007.

[14] U. Eminoglu, M. H. Hocaoglu, "A Voltage Stability Index for Radial Distribution Networks", UPEC 2007 - 408

[15] Partha Kaya, Sayonsom Chanda, C. K. Chanda, "Determination of Voltage Stabilityin Distribution Network Using ANN Technique", International Journal on Electrical Engineering and Informatics - Vol. 4, No. 2, July 2012.

[16] Jen-Hao T, “A Direct Approach for Distribution System Load Flow Solutions”, IEEE Trans. on Power Delivery, 2003, vol.18, no.3, pp. 882-887.

[17] J. Kennedy and R. Eberhart. Swarm Intelligence. Morgan Kaufmann Publishers, Inc., San Francisco, CA, 2001.

[18] Kennedy, J.; Eberhart, R.C. "A discrete binary version of the particle swarm algorithm", IEEE International Conference on Systems, Man, and Cybernetics, 1997.

[19] Mojtaba Ahmadieh Khanesar., Mohammad Teshnehlab., Mahdi Aliyari Shoorehdeli., "A Novel Binary Particle Swarm Optimization", Proceedings of the 15th Mediterranean Conference on Control and Automation, July 2007, Athens-greece.

[20] Md. Imran Azim, Md. Fayzur Rahman, "Genetic Algorithm Based Reactive Power Management by SVC", International Journal of Electrical and Computer Engineering, Vol. 4, No. 2, April 2014, pp. 200 206. 